\title{
Induction and binary expression of LTP/LTD in a minimal model of the CaMKII system Michael Graupner*1,2 and Nicolas Brunel ${ }^{1}$
}

\author{
Address: ${ }^{1}$ Laboratoire de Neurophysique et Physiologie, CNRS UMR 8119, Université Paris Descartes - Paris V, 45, rue des Saints Pères, 75270 \\ Paris Cedex 06, France and 2Max-Planck-Institut für Physik komplexer Systeme, Nötnitzer Straße 38, 01187 Dresden, Germany \\ Email: Michael Graupner* - michael.graupner@univ-paris5.fr \\ * Corresponding author
}

from Sixteenth Annual Computational Neuroscience Meeting: CNS*2007

Toronto, Canada. 7-12 July 2007

Published: 6 July 2007

BMC Neuroscience 2007, 8(Suppl 2):P94 doi: I0.I |86/I47|-2202-8-S2-P94

(C) 2007 Graupner and Brunel; licensee BioMed Central Ltd.

The calcium/calmodulin-dependent protein kinase II (CaMKII) plays a key role in the induction of long-term post-synaptic modifications following synaptic activation. Experiments suggest that these long-term synaptic changes are all-or none switch-like events between discrete states [1]. The biochemical network involving CaM$\mathrm{KII}$ and its regulating protein signaling cascade has been hypothesized to durably maintain the evoked synaptic state in the form of a bistable switch $[2,3]$. However, it is still unclear whether different experimental LTP/LTD protocols lead to corresponding transitions between the two states in models of such a network. Furthermore, the biochemical mechanisms and signaling cascades giving rise to the non-linearities exhibited during LTP/LTD induction remain elusive.

Starting from a detailed biochemical model, a minimal model describing the CaMKII phosphorylation (activation) level is presented which preserves the features of a comprehensive description. CaMKII autophosphorylation is governed by calcium/calmodulin binding and is a highly cooperative process. CaMKII dephosphorylation is mediated by protein phosphatase 1 whose activity is indirectly regulated by a calcium-dependent balance of kinase (protein kinase A) and phosphatase (calcineurin) activity. These two competing effects are implemented via phosphorylation- and dephosphorylation rates changing the CaMKII phosphorylation level and are realized as simple step functions activating above different calcium levels.
The model retains previous results [2,3], two stable states of CaMKII phosphorylation exist at resting intracellular calcium concentrations. With an appropriate positioning of the de-/phosphorylation thresholds, high calcium transients can switch the system from the weakly-(DOWN) to the highly-phosphorylated (UP) state of the CaMKII ( similar to a LTP event) and intermediate $\mathrm{Ca}(2+)$ concentrations can lead to switching from the UP to the DOWN state (similar to a LTD event). As a basic principle, this can be achieved if the CaMKII dephosphorylation activates at lower $\mathrm{Ca}(2+)$ levels than phosphorylation. This simple approach allows us to address whether or not a read-out system using the calcium level as the sole input signal can account for the non-linearities exhibited during LTP/LTD induction. It is shown that this simple realization of the CaMKII system can qualitatively reproduce experimental plasticity results in response to spike-timing dependent plasticity (STDP) protocols (spike-pairs and -triplets), presynaptic stimulation protocols and pairing protocols. Our investigations show that a minimal model of the CaMKII protein network can account for both induction (through LTP/LTD-like transitions) and storage (due to its bistability) of synaptic changes. However, we suggest that the dynamics of the global calcium time course play a crucial role for the sign of synaptic changes alongside the crosstalk between signaling cascades that include the one considered here. 


\section{References}

I. O'Connor DH, Wittenberg GM, Wang SS-H: Graded bidirectional synaptic plasticity is composed of switch-like unitary events. Proc Natl Acad Sci USA 2005, 102:9679-84.

2. Zhabotinsky AM: Bistability in the $\mathbf{C a}(2+) / c a l m o d u l i n-d e p e n d-$ ent protein kinase-phosphatase system. Biophys J 2000, 79:22II-222I.

3. Okamoto H, Ichikawa K: Switching characteristics of a model for biochemical-reaction networks describing autophosphorylation versus dephosphorylation of $\mathrm{Ca}(2+) / c a l m o d u l i n-$ dependent protein kinase II. Biol Cybern 2000, 82:35-47.

Publish with Bio Med Central and every scientist can read your work free of charge

"BioMed Central will be the most significant development for disseminating the results of biomedical research in our lifetime. " Sir Paul Nurse, Cancer Research UK

Your research papers will be:

- available free of charge to the entire biomedical community

- peer reviewed and published immediately upon acceptance

- cited in PubMed and archived on PubMed Central

- yours - you keep the copyright

Submit your manuscript here:

http://www.biomedcentral.com/info/publishing_adv.asp 